Proceedings of the

International Geometry Center

Vol. 12 , no. 4 (2019) pp. $69-88$

\title{
On semiconvexity of open sets with smooth boundary in the plane
}

\author{
Tetiana Osipchuk
}

\begin{abstract}
We study properties of classes of generalized convex sets in the plane known as 1-semiconvex and weakly 1-semiconvex. It is proved that an open, weakly 1-semiconvex but not 1-semiconvex set with smooth boundary in the plane consists of at least four connected components.
\end{abstract}

Анотація. В даній роботі вивчаються властивості класів узагальнено опуклих множин на площині, які називаються 1-напівопуклими та слабко 1-напівопуклими.

Відкрита множина багатовимірного дійсного евклідового простору $\mathbb{R}^{n}$ називається 1-напівопуклою, якщо для кожної точки із доповнення цієї множини до всього простору існує промінь, який починається в цій точці і не перетинає задану множину. Відкрита множина простору $\mathbb{R}^{n}$ називається слабко 1-напівопуклою, якщо для кожної точки межі множини існує промінь, який починається в цій точці та не перетинає задану множину. Ці поняття були введені Юрієм Борисовичем Зелінським. Усяка відкрита 1-напівопукла множина очевидно є слабко 1-напівопуклою. Ю. Б. Зелінський показав, що зворотне твердження є невірним. Виявилось, що клас відкритих множин на площині, які є слабко 1-напівопуклими але не 1-напівопуклими, є досить широким і кожна множина 3 цього класу є незв'язною і складається не менше ніж з трьох компонент зв'язності.

Дана робота присвячена переважно дослідженню нових властивостей слабко 1-напівопуклих але не 1-напівопуклих множин на площині. Тут вони, для зручності, називаються множинами, що мають $Z$-властивість.

The work is dedicated to the memory of Yu. B. Zelinskii. The author expresses her gratitude to Professors Sergiy Maksymenko, Sergiy Plaksa, Oleksandr Bakhtin, Serhii Gryshchuk, Ruslan Salimov, Vitalii Shpakivskyi for valuable advice in planning the study and recommendations for the design of the paper. The author also is grateful to translators Julia Sheichenko and Anna Sheichenko who assisted in the preparation of the manuscript before being sent to the publisher. The author expresses her gratitude to all anonymous Referees of the paper for their comments.

2010 Mathematics Subject Classification: 32F17, 52A30

$U D C 514.172$

Keywords: Convex set, open set, smooth boundary, real Euclidean space 
Основний результат роботи наступний: доведено, що відкрита множина з гладкою межею на площині, яка має $Z$-властивість, складається щонайменше з чотирьох компонент зв'язності.

Аннотация. В данной работе изучаются свойства классов обобщенно выпуклых множеств на плоскости, которые называются 1-полувыпуклыми и слабо 1-полувыпуклыми. В частности, доказано, что открытое слабо 1-полувыпуклое, но не 1-полувыпуклое множество с гладкой границей на плоскости состоит не менее чем из четырёх компонент связности.

\section{INTRODUCTION}

A class of $m$-semiconvex sets is one of the classes of generalized convex sets. A semiconvexity notion was proposed by Yu. Zelinskii [9] and it was used in the formulation of a generalization of shadow problem. The shadow problem was proposed by G. Khudaiberganov [4] in 1982. It requires to find the minimal number of open (closed) balls in the real Euclidean space $\mathbb{R}^{n}$ that are pairwise disjoint, centered on a sphere $S^{n-1}$ (see [6]), do not contain the sphere center, and such that any straight line passing through the sphere center intersects at least one of the balls.

To formulate the generalized shadow problem, let us give at first the following definitions which we also use in our investigation.

Each $m$-dimensional affine subspace of the space $\mathbb{R}^{n}, 0 \leqslant m<n$, is called an $m$-dimensional plane.

Definition 1.1. One of two parts of an $m$-dimensional plane, $m \geqslant 1$, of the space $\mathbb{R}^{n}, n \geqslant 2$, into which it is divided by its any $(m-1)$-dimensional plane (herewith, the points of the $(m-1)$-dimensional plane are included) is said to be an m-dimensional half-plane.

For instance, the 1-dimensional half-plane is a ray, the 2-dimensional half-plane is a half-plane, etc.

Definition 1.2. ([7]) A subset $E \subset \mathbb{R}^{n}$ is called m-semiconvex with respect to a point $x \in \mathbb{R}^{n} \backslash E, 1 \leqslant m<n$, if there exists an $m$-dimensional half-plane $L$ such that $x \in L$ and $L \cap E=\varnothing$.

A subset $E \subset \mathbb{R}^{n}$ is called $m$-semiconvex, $1 \leqslant m<n$, if it is $m$ semiconvex with respect to every point $x \in \mathbb{R}^{n} \backslash E$.

One can easily see that both definitions satisfy the axiom of convexity: the intersection of each subfamily of these sets also satisfies the definition. Thus, for any subset $E \subset \mathbb{R}^{n}$ we can consider the minimal $m$-semiconvex set containing $E$. This set is called the $m$-semiconvex hull of $E$. 
The generalized shadow problem requires to find the minimum number of pairwise disjoint closed (open) balls in $\mathbb{R}^{n}$ (centered on a sphere $S^{n-1}$ and whose radii are smaller than the radius of the sphere) such that any ray starting at the center of the sphere necessarily intersects at least one of these balls.

In the terms of $m$-semiconvexity this problem can be reformulated as follows: what is the minimum number of pairwise disjoint closed (open) balls in $\mathbb{R}^{n}$ whose centers are located on a sphere $S^{n-1}$ and the radii are smaller than the radius of this sphere such that the center of the sphere belongs to the 1-semiconvex hull of the family of these balls?

In [9] the generalized shadow problem is solved as $n=2$ and only the sufficient number of balls is indicated for $n=3$.

In the 60's L. Aizenberg and A. Martineau proposed their notions of a linearly convex set in the multi-dimensional complex space $\mathbb{C}^{n}$. The first author considered domains and their closures and used boundary points of the domains in his definition $[1,2]$. The second author used all points of the complement to a subset of the space $\mathbb{C}^{n}$, [5]. If one uses these definitions not only for domains and compact sets, then Aizenberg's definition isolates one connected component of a set which is linearly convex in the sense of Martineau.

Guided by similar ideas Yu. Zeliskii suggested to distinguish $m$-semiconvex and weakly $m$-semiconvex sets.

We will use the following standard notations. For a subset $G \subset \mathbb{R}^{n}$ let $\bar{G}$ be its closure, Int $G$ be its interior, and $\partial G=\bar{G} \backslash \operatorname{Int} G$ be its boundary.

Say that a set $A$ is approximated from the outside by a family of open sets $A_{k}, k=1,2, \ldots$, if $\bar{A}_{k+1}$ is contained in $A_{k}$, and $A=\underset{k}{\cap} A_{k}$ (see [2]).

Definition 1.3. ([8]) An open subset $G \subset \mathbb{R}^{n}$ is called weakly m-semiconvex, $1 \leqslant m<n$, if it is $m$-semiconvex with respect to any point $x \in \partial G$. A subset $E \subset \mathbb{R}^{n}$ is called weakly $m$-semiconvex if it can be approximated from the outside by a family of open weakly $m$-semiconvex sets.

Thus, each weakly $m$-semiconvex set $A$ is either open or closed. Among closed weakly $m$-semiconvex sets there also are sets with empty interior:

$$
A=\bar{A}=\bar{A} \backslash \operatorname{Int} A=\partial A .
$$

Theorem 1.4. ([8]) Let $E \subset \mathbb{R}^{2}$ be an open, weakly 1-semiconvex and not 1-semiconvex subset. Then $E$ is disconnected.

The formulation of the following theorem is equivalent to Theorem 1.4 but will be also in use. 
Theorem 1.5. Let $E \subset \mathbb{R}^{2}$ be open, connected and weekly 1-semiconvex. Then $E$ is 1-semiconvex.

The maximal connected subsets of a topological space $A$ are called connected components (components) of $A$ (see [2]).

In [8] it was constructed the example of an open, weakly 1-semiconvex, and not 1-semiconvex set (see Figure 2.3b). It was also conjectured that every open, weakly 1-semiconvex, and not 1-semiconvex set consists of at least three components. The latter statement was proved in [3].

Theorem 1.6. ([3]) Let $E \subset \mathbb{R}^{2}$ be an open, weakly 1-semiconvex, and not 1-semiconvex subset. Then $E$ consists of at least three connected components.

We say that a component $A$ of an open, bounded subset of the plane has smooth boundary if $\partial A$ is the image of a $C^{1}$-embedding of the unit circle. We say that an open, bounded subset of the plane has smooth boundary if each of its components has smooth boundary.

The present paper continues the research of Yu. Zelinskii by investigating properties of 1-semiconvex and weakly 1-semiconvex open sets with smooth boundary in the plane.

For the convenience, we give the following

Definition 1.7. We say that a set in the plane has $Z$-property if the set is weakly 1-semiconvex and not 1-semiconvex.

The main result of thee present paper is the following

Theorem 1.8. Suppose that an open, bounded subset $E \subset \mathbb{R}^{2}$ with smooth boundary has $Z$-property. Then $E$ consists of at least four components.

\section{Auxiliary Results}

In what follows, unless otherwise is specified, a point of the space $\mathbb{R}^{2}$ will be denoted by a small or capital Latin letter and the ray starting at that point will be denoted by a small Greek letter with the index denoting this point. The straight segment between points $x, y \in \mathbb{R}^{n}$ will be denoted by $x y$ and the distance between them will be denoted by $|x-y|$.

Let us provide several auxiliary statements.

Proposition 2.1. If an open subset $E \subset \mathbb{R}^{n}$ is $m$-semiconvex, then it is weakly m-semiconvex.

Proposition 2.2. There exist sets in the plane having Z-property. 
In the following example we present an open subset of $\mathbb{R}^{2}$ with $Z$ property.

Example 2.3. Figure 2.1a) shows a set $E$ consisting of four open rectangles with a common rays passing through their boundaries. By the construction, for any boundary point of $E$ there exists a ray that does not intersect $E$, while for any point of the interior of the rhombus abcd such a ray can not be found.

Similarly, in Figure 2.1b), the set consisting of three open components is weakly 1-semiconvex. On the other hand it is not 1-semiconvex, since each ray $\eta_{x}$ starting at each point $x$ of the interior of the triangle $a b c$ intersects that set.
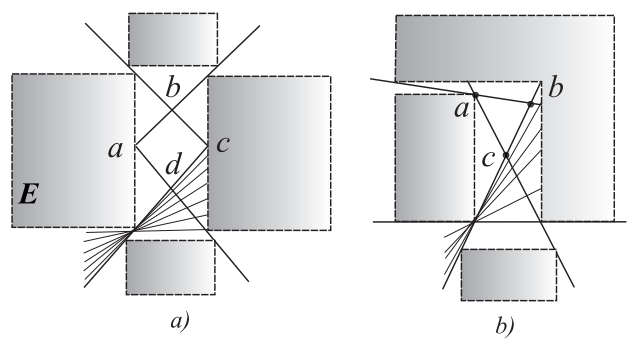

FiguRE 2.1.

Example 2.4. An example of open set with smooth boundary having $Z$ property can be obtained by replacing the rectangles from Figure 2.1a) with open sets having smooth boundaries tangent to the same rays and such that their union is weakly 1-semiconvex. For instance, this property holds for a system of four open disks shown in Figure 2.2a).

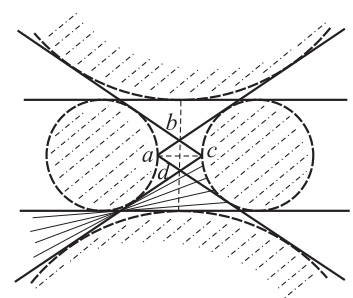

a)

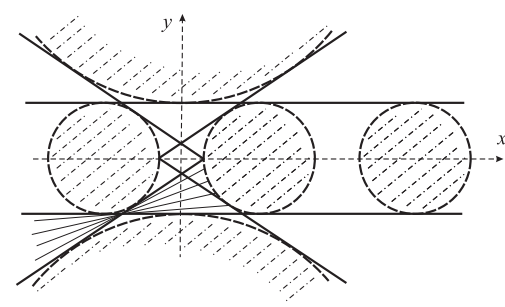

b)

FiguRE 2.2.

Suppose that two disks of that system have the same radii $r$ and their centers belong to the axis $O x$ and are symmetric with respect to the origin $O$, see Figure 2.2b). Assume that the other two disks have radii $R$ and 
their centers belong to the axis $O y$. If we place the center of the fifth open disk with radius $r$ at the point $(R+r, 0)$, then the union of those five open disks will have $Z$-property. Adding more disks of radius $r$ and centers on the axis $O x$ in the positive direction, we will get an open set with smooth boundary having $Z$-property and consisting of any finite or even countable number of components.

Further it will be shown that in the example of a set having $Z$-property and consisting of three components it is not possible to replace the components with those that have smooth boundary while maintaining $Z$-property.

The set of all points of rays starting at a point $x \in \mathbb{R}^{n} \backslash A$ and passing through a subset $A \subset \mathbb{R}^{n}$ is called the cone over the set $A$ with respect to the point $x$ and is denoted by $\mathrm{C}_{x} A$. The boundary of $\mathrm{C}_{x} A$ consists of rays which are called boundary rays. The boundary rays of $\mathrm{C}_{x} A$ are called supporting for the set $A$ (with respect to the point $x$ ). We suppose that $x \notin \mathrm{C}_{x} A$ whenever $A$ is open and $x \in \mathrm{C}_{x} A$ otherwise.

It can be proved that if $A$ is bounded and open (closed), then $\mathrm{C}_{x} A$ is open (closed) as well and $\mathrm{C}_{x} \bar{A} \equiv \overline{\mathrm{C}_{x} A}$.

Lemma 2.5. A ray $\gamma_{x}$ is supporting for an open bounded subset $E \subset \mathbb{R}^{n}$ iff the following conditions are satisfied:

1) $\gamma_{x} \cap \partial E \neq \varnothing$

2) $\gamma_{x} \cap E=\varnothing$.

Proof. Necessity. Suppose $a^{\prime} \in \gamma_{x} \cap E \neq \varnothing$, then there exists a neighborhood $U\left(a^{\prime}\right) \subset E$ of $a^{\prime}$ such that $\gamma_{x} \subset \mathrm{C}_{x}\left(U\left(a^{\prime}\right)\right) \subset \mathrm{C}_{x} E$ and the ray $\gamma_{x}$ is not the boundary one. If $\gamma_{x} \cap \partial E=\varnothing$, then $\gamma_{x} \cap \bar{E}=\varnothing$, i.e $\gamma_{x} \subset \mathbb{R}^{n} \backslash \mathrm{C}_{x} \bar{E} \equiv \mathbb{R}^{n} \backslash \overline{\mathrm{C}_{x} E}$ which implies that the ray $\gamma_{x}$ is also not the boundary one.

Sufficiency. Consider the cone $\mathrm{C}_{x} E$. Since $E$ is open, $\mathrm{C}_{x} E$ is open as well. We need to show that the ray $\gamma_{x}$ satisfying conditions 1) and 2) coincides with one of boundary rays of $\mathrm{C}_{x} E$. If $\gamma_{x} \subset \mathbb{R}^{n} \backslash \overline{\mathrm{C}_{x} E}$, then condition 1) of the lemma is not true. On the other hand, if $\gamma_{x} \subset \mathrm{C}_{x} E$, then $\gamma_{x} \cap E \neq \varnothing$, by the definition of $\mathrm{C}_{x} E$, which contradicts to condition 2). Thus, $\gamma_{x} \subset \partial \mathrm{C}_{x} E$.

Lemma 2.6. For an open connected weakly 1-semiconvex subset $E \subset \mathbb{R}^{2}$ there exists at least one but no more than two supporting rays starting at some point $x \in \mathbb{R}^{2} \backslash E$.

Proof. By Theorem 1.5 the set $E$ is 1-semiconvex. Thus for any point $x \in \mathbb{R}^{2} \backslash E$ there exists a ray $\gamma_{x}$ such that $\gamma_{x} \cap E=\varnothing$. Since $E$ is not empty, open and connected, $\mathrm{C}_{x} E$ is a plane angle $\angle \alpha>0$ not containing its sides. 
If $\gamma_{x}$ is unique, i.e. $\gamma_{x} \equiv \mathbb{R}^{2} \backslash \mathrm{C}_{x} E$, then $\angle \alpha=2 \pi$ and $\gamma_{x}$ is its unique side. On the other hand, if $\gamma_{x} \subsetneq \mathbb{R}^{2} \backslash \mathrm{C}_{x} E$, then $0<\angle \alpha<2 \pi$ with two sides.

An example of open, connected, and weakly 1-semiconvex set $E$ for which there exists a point $x \in \mathbb{R}^{2} \backslash E$ admitting a unique supporting ray $\xi_{x}$ is shown in Figure 2.3a).

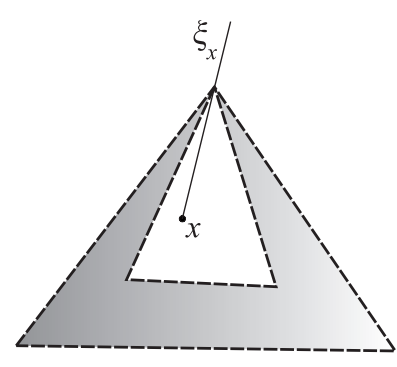

a)

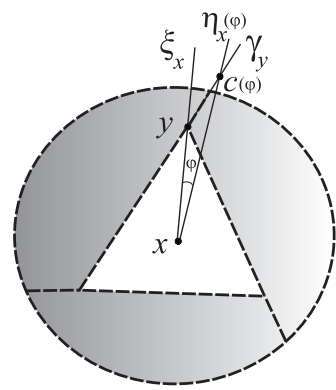

b)

FiguRe 2.3 .

Definition 2.7. A point $x \in \mathbb{R}^{n} \backslash A$ is called a point of m-nonsemiconvexity of a subset $A \subset \mathbb{R}^{n}$ if there is no $m$-dimensional half-plane that has $x$ on its boundary and does not intersect $A$.

Lemma 2.8. Suppose that an open subset $E=\bigcup_{j=1}^{k} E_{j} \subset \mathbb{R}^{2}, k \geqslant 3, k \in \mathbb{N}$, where $E_{j}$ are its components, has $Z$-property. Let also $x$ be a point of 1 nonsemiconvexity of $E$. Then each component $E_{j}$ has exactly two supporting rays starting at $x$.

Proof. Since $E$ has $Z$-property, it follows that each of its 1-nonsemiconvexity point $x$ belongs to $\mathbb{R}^{2} \backslash \bar{E}$.

By Lemma 2.8 each component $E_{j}$ has either one or two supporting rays starting at $x$. Suppose some component $E_{j^{1}}, j^{1} \in\{1,2, \ldots, k\}$, has a unique supporting ray $\xi_{x}$. Since $E$ is not 1-semiconvex, it follows that $\xi_{x} \cap\left(E \backslash E_{j^{1}}\right) \neq \varnothing$. Let $\xi_{x}^{\prime}$ be the ray complementary to $\xi_{x}$ and $y$ be the nearest point of $\partial E_{j^{1}}$ to $x$ along the ray $\xi_{x}^{\prime}$.

Notice that any ray starting at $y$ and not containing the ray $\xi_{x}$ intersects $E_{j^{1}}$. Indeed, suppose there exists a ray $\gamma_{y}$ not containing the ray $\xi_{x}$ and such that $\gamma_{y} \cap E_{j^{1}}=\varnothing$. Let $\xi_{y}$ be the ray starting at $y$ and containing $\xi_{x}$. Then the polygonal chain $\gamma_{y} \cup\{y\} \cup \xi_{y}$ cuts the plane into two open components (parts). Since $E_{j^{1}} \cap\left(\gamma_{y} \cup\{y\} \cup \xi_{y}\right)=\varnothing$ and $E_{j^{1}}$ is connected, it is completely contained in one of those parts. On the other hand, there 
exists a ray $\eta_{x}$ contained in the other part, which gives $\eta_{x} \cap E_{j^{1}}=\varnothing$. Since $\eta_{x}$ differs from $\xi_{x}$, it is clear that $\xi_{x}$ is not a unique supporting ray for $E_{j^{1}}$ with respect to the point $x$. This contradicts to our assumption that $E_{j 1}$ has a unique supporting ray.

Furthermore, since $\xi_{x}$ intersects $E \backslash E_{j^{1}}$, the ray $\xi_{y} \supset \xi_{x}$ also intersects $E \backslash E_{j^{1}}$. Thus, $y \in \partial E$ is a point of 1 -nonsemiconvexity of $E$, which contradicts to weak 1-semiconvexity of $E$ and none of the components of $E$ have a unique supporting ray.

Definition 2.9. Let $A \subset \mathbb{R}^{n}, A_{1}$ be a component of $A$, and $x \in \mathbb{R}^{n} \backslash(\bar{A})$. A ray $\xi_{x}$ is called inner supporting for the set $A$ with respect to $A_{1}$, whenever $\xi_{x}$ is supporting for $A_{1}, \xi_{x} \cap\left(A \backslash A_{1}\right) \neq \varnothing$, and there exists a point $a \in \xi_{x} \cap \partial A_{1}$ such that $|a-x|<|b-x|$ for any point $b \in \xi_{x} \cap\left(A \backslash A_{1}\right)$.

Definition 2.10. Let $A \subset \mathbb{R}^{n}$ and $x \in \mathbb{R}^{n} \backslash(\bar{A})$. A ray $\xi_{x}$, is called inner supporting for $A$ if there exists a component $A_{1}$ of $A$ such that $\xi_{x}$ is inner supporting for $A$ with respect to $A_{1}$.

Lemma 2.11. Let $E_{1}, E_{2}$ be two components of a subset $E \subset \mathbb{R}^{n}$ and let $x \in \mathbb{R}^{n} \backslash \bar{E}$. If a ray $\xi_{x}$ is inner supporting for $E_{1} \cup E_{2}$ and

$$
\xi_{x} \cap\left(E \backslash\left(E_{1} \cup E_{2}\right)\right)=\varnothing,
$$

then $\xi_{x}$ is inner supporting for $E$.

Proof. Without loss of generality, suppose that $\xi_{x}$ is inner supporting for the set $E_{1} \cup E_{2}$ with respect to $E_{1}$. Then $\xi_{x}$ is supporting for $E_{1}$ and has the following properties:

a) $\xi_{x} \cap E_{2} \neq \varnothing$,

b) there exists a point $a \in \xi_{x} \cap \partial E_{1}$ such that $|a-x|<|b-x|$ for every $b \in \xi_{x} \cap E_{2}$ by Definition 2.9.

Condition a) together with condition $E_{2} \subset E \backslash E_{1}$ gives $\xi_{x} \cap\left(E \backslash E_{1}\right) \neq \varnothing$. Since $\xi_{x} \cap\left(E \backslash\left(E_{1} \cup E_{2}\right)\right)=\varnothing$, we have

$$
\xi_{x} \cap E_{2} \equiv \xi_{x} \cap\left(\left(E \backslash\left(E_{1} \cup E_{2}\right)\right) \cup E_{2}\right) \equiv \xi_{x} \cap\left(E \backslash E_{1}\right),
$$

which together with condition b) gives $|a-x|<|b-x|$ for the point $a \in \xi_{x} \cap \partial E_{1}$ and for any point $b \in \xi_{x} \cap\left(E \backslash E_{1}\right)$. Thus, $E_{1}$ is a component of $E$ such that $\xi_{x}$ is an inner supporting ray for $E$ with respect to $E_{1}$.

Lemma 2.12. Let $E=\bigcup_{j=1}^{k} E_{j} \subset \mathbb{R}^{2}, 3 \leqslant k<\infty, k \in \mathbb{N}$, be an open bounded set having $Z$-property, where $E_{j}$ are its components. Let $x \in \mathbb{R}^{2} \backslash \bar{E}$ be a point of 1-nonsemiconvexity of $E, y \in \partial E$ the nearest point of $\partial E$ to $x$ along some ray $\eta_{x}$, and $\gamma_{y}$ any ray that does not intersect $E$. 
Then there exists an inner supporting ray $\xi_{x}$ for $E$ with respect to some component $E_{j^{0}}, j^{0} \in\{\overline{1, k}\}$, such that

1) $\xi_{x} \cap \gamma_{y}=c \neq \varnothing$

2) $|a-x| \leqslant|c-x|$ for any point $a \in \xi_{x} \cap \partial E_{j^{0}}$;

3) $E \cap \operatorname{Int}(\triangle x y c)=\varnothing$.

Proof. Since $x$ is a point of 1-nonsemiconvexity of $E, \gamma_{y}$ does not lie on the straight line that contains $\eta_{x}$.

Choose the polar coordinate system $(\varphi, \rho)$ in $\mathbb{R}^{2}$ in which $x$ is the pole, the ray $\eta_{x}=\eta_{x}(0)$ is the polar axis, $\eta_{x}(\varphi)$ is a ray starting at $x$ and constituting an angle $\varphi$ with ray $\eta_{x}$, and a positive angular coordinate $\varphi$ is determined by a ray starting at $x$ and intersecting ray $\gamma_{y}$.

Let $\eta_{x}(\phi), 0<\phi<\pi$, be the ray that is parallel to $\gamma_{y}$. Then rays $\eta_{x}(\varphi), 0<\varphi<\phi$, intersect $\gamma_{y}$. We will also use the following notations: $c(\varphi)=\eta_{x}(\varphi) \cap \gamma_{y}$ and $x c(\varphi)$ is the interval between points $x$ and $c(\varphi)$.

Let $\Phi$ be the set of all $\varphi \in(0, \phi)$ such that $x c(\varphi) \cap E \neq \varnothing$. Then $\Phi$ is non-empty. Indeed, since $\eta_{x}(\phi) \cap E \neq \varnothing$ and $E$ is open, there exists $\varepsilon>0$ small enough and such that $x c(\phi-\varepsilon) \cap E \neq \varnothing$.

Let $J \subseteq\{1,2, \ldots, k\}$ be the set of all indexes $j \in\{1,2, \ldots, k\}$ such that there exists $\varphi \in \Phi$ for which $x c(\varphi) \cap E_{j} \neq \varnothing$. Let also $\Phi_{j} \subseteq \Phi, j \in J$, be the set of all $\varphi \in \Phi$ with $x c(\varphi) \cap E_{j} \neq \varnothing$.

Put $\varphi_{j}=\inf \Phi_{j}, j \in J$. Then it is clear that $\varphi_{j} \in[0, \phi)$ and

a) $x c\left(\varphi_{j}\right) \cap \partial E_{j} \neq \varnothing$,

b) $x c\left(\varphi_{j}\right) \cap E_{j}=\varnothing$,

c) $x c\left(\varphi_{j}+\varepsilon\right) \cap E_{j} \neq \varnothing$, for $\varepsilon>0$ small enough.

This implies that $E_{j} \cap \operatorname{Int}\left(\triangle x y c\left(\varphi_{j}\right)\right)=\varnothing, j \in J$.

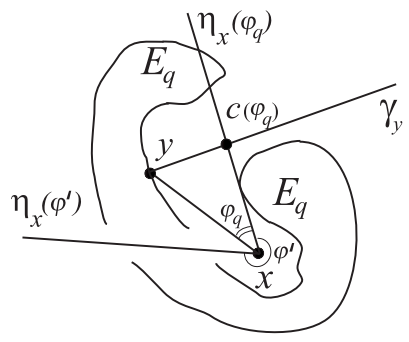

a)

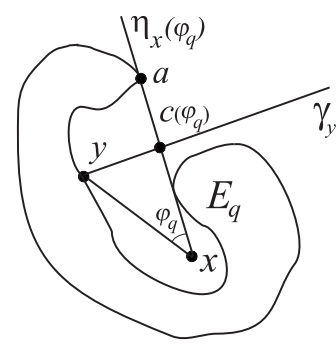

b)

FiguRE 2.4.

We claim that if $\eta_{x}\left(\varphi_{q}\right) \cap E_{q} \neq \varnothing$ for some $q \in J$, then $E_{q}$ does not have supporting rays starting at $x$, see Figure 2.4a). Indeed, suppose $\eta_{x}\left(\varphi^{\prime}\right)$, $\varphi^{\prime} \neq \varphi_{q}$, is supporting for $E_{q}$. Then $\eta_{x}\left(\varphi^{\prime}\right) \cap E_{q}=\varnothing$. Let $\gamma_{c\left(\varphi_{q}\right)}$ be the ray 
starting at point $c\left(\varphi_{q}\right)$ and lying on ray $\gamma_{y}$. If $\eta_{x}\left(\varphi^{\prime}\right) \cap \gamma_{c\left(\varphi_{q}\right)}=\varnothing$, then the polygonal chain

$$
L:=\eta_{x}\left(\varphi^{\prime}\right) \cup\{x\} \cup x c\left(\varphi_{q}\right) \cup\left\{c\left(\varphi_{q}\right)\right\} \cup \gamma_{c\left(\varphi_{q}\right)}
$$

cuts the plane into two open components. Herewith, $L \cap E_{q}=\varnothing$. One of the components of $\mathbb{R}^{2} \backslash L$ contains a part of the ray $\eta_{x}\left(\varphi_{q}\right)$ intersecting $E_{q}$. Since $E_{q}$ is connected, it is entirely contained in this component. On the other hand, since the interval $x c(\varphi), \varphi_{q}<\varphi<\phi$, is contained in the second component of $\mathbb{R}^{2} \backslash L$ and $x c\left(\varphi_{q}+\varepsilon\right) \cap E_{q} \neq \varnothing$, for $\varepsilon>0$ small enough, $E_{q}$ is contained in the second component. We have reached a contradiction.

If $\eta_{x}\left(\varphi^{\prime}\right) \cap \gamma_{c\left(\varphi_{q}\right)} \neq \varnothing$, then the polygonal chain $L$ cuts the plane into three open components. The component of $\mathbb{R}^{2} \backslash L$ containing the part of the ray $\eta_{x}\left(\varphi_{q}\right)$ intersecting $E_{q}$ contains $E_{q}$ as well. Moreover, the component of $\mathbb{R}^{2} \backslash L$ bounded by the triangle generated by the intersection of rays $\eta_{x}\left(\varphi^{\prime}\right)$, $\gamma_{c\left(\varphi_{q}\right)}$, and $\eta_{x}\left(\varphi_{q}\right)$ also contains $E_{q}$, since $x c\left(\varphi_{q}+\varepsilon\right) \cap E_{q} \neq \varnothing$, for $\varepsilon>0$ small enough. This contradicts to the fact that $E_{q}$ is connected. Thus, our assumption is incorrect and $E_{q}$ does not have supporting rays starting at $x$.

Similarly, it can be proved that if $\eta_{x}\left(\varphi_{q}\right) \cap E_{q}=\varnothing$ but there exists a point $a \in \eta_{x}\left(\varphi_{q}\right) \cap \partial E_{q}$ such that $|a-x|>\left|c\left(\varphi_{q}\right)-x\right|$ for some $q \in J$, then $E_{q}$ has a unique supporting ray starting at $x$ coinciding with $\eta_{x}\left(\varphi_{q}\right)$ (see Figure $2.4 \mathrm{~b}$ ).

The cases when component $E_{q}$ does not have supporting rays or has a unique supporting ray contradict to Lemma 2.8. Then by Lemma 2.5, for any $j \in J$ the ray $\eta_{x}\left(\varphi_{j}\right)$ is supporting for $E_{j}$ and has the following properties:

a) $\eta_{x}\left(\varphi_{j}\right) \cap \gamma_{y}=c\left(\varphi_{j}\right)$;

b) $|a-x| \leqslant\left|c\left(\varphi_{j}\right)-x\right|$ for any point $a \in \eta_{x}\left(\varphi_{j}\right) \cap \partial E_{j}$;

c) $E_{j} \cap \operatorname{Int}\left(\triangle x y c\left(\varphi_{j}\right)\right)=\varnothing$.

Thus, conditions 1) and 2) of our lemma are fulfilled for any ray $\eta_{x}\left(\varphi_{j}\right)$, $j \in J$.

To finish the proof, we need only to show that among rays $\eta_{x}\left(\varphi_{j}\right), j \in J$, there is the one that is inner supporting for $E$ and satisfying the lemma condition 3). Consider the angle $\varphi_{j^{0}}:=\min _{j} \varphi_{j}, j^{0} \in J$, and note that by the constructions $E \cap \triangle \operatorname{Int}\left(x y c\left(\varphi_{j^{0}}\right)\right)=\varnothing$. Thus, condition 3) holds for the ray $\eta_{x}\left(\varphi_{j}\right)$. Since $x$ is a point of 1-nonsemiconvexity of $E$, it follows that $\eta_{x}\left(\varphi_{j^{0}}\right) \cap\left(E \backslash E_{j^{0}}\right) \neq \varnothing$. It then follows from properties 1) and 2), that $|a-x|<|b-x|$ for any point $a \in \eta_{x}\left(\varphi_{j^{0}}\right) \cap \partial E_{j^{0}}$ and any point $b \in \eta_{x}\left(\varphi_{j^{0}}\right) \cap\left(E \backslash E_{j^{0}}\right)$. Thus, $\xi_{x}:=\eta_{x}\left(\varphi_{j^{0}}\right)$ is an inner supporting ray of $E$ with respect to $E_{j 0}$ satisfying the conditions 1)-3) of the lemma. 
Remark 2.13. Notice that if $\partial E$ is not smooth at $y$, then the inner supporting ray $\xi_{x}$ can coincide with the ray $\eta_{x}$. In this case the points $c$ and $y$ coincide and $\triangle x y c$ degenerates into the interval $x y$, see Figure $2.3 \mathrm{~b}$ ). Then all rays $\eta_{x}(\varphi)$ which are close enough to $\xi_{x} \equiv \eta_{x}$ and intersect ray $\gamma_{y}$ must also intersect the component $E_{j^{0}}$.

If $\partial E$ is smooth, then $\triangle x y c$ is non-degenerate, due to the fact that $y$ is the nearest to $x$ along $\eta_{x}$.

Definition 2.14. We say that a subset $A \subset \mathbb{R}^{n}$ is projected from a point $x \in \mathbb{R}^{n}$ on a subset $B \subset \mathbb{R}^{n}$ if any ray starting at point $x$ and intersecting $A$ intersects $B$ as well.

Lemma 2.15. Suppose an open subset $E \subset \mathbb{R}^{2}$ has $Z$-property and consists of three components. Then none of its components is projected on the union of the others from a point of 1-nonsemiconvexity of E.

Proof. Let $E_{1}, E_{2}, E_{3}$ be connected components of $E$ and $x \in \mathbb{R}^{2} \backslash \bar{E}$ be a point of 1-nonsemiconvexity of $E$. Without loss of generality, suppose that $E_{1}$ is projected from $x$ on $E_{2} \cup E_{3}$, see Figure 2.5. Then the set, consisting only of components $E_{2}, E_{3}$, has $Z$-property, which contradicts to Theorem 1.6.

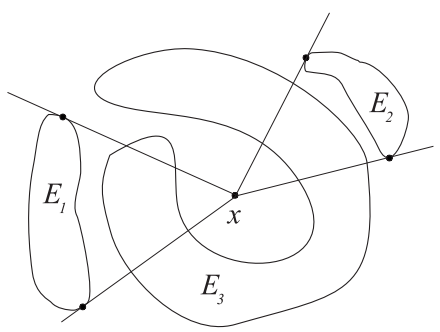

a)

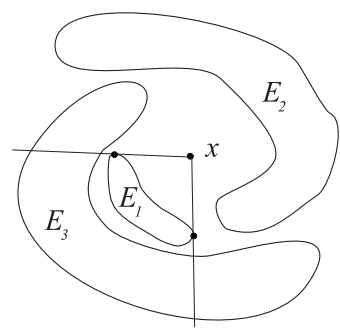

b)

FIGURE 2.5.

Let $E \subset \mathbb{R}^{2}$ be an open subset and $\gamma$ a straight line passing through some boundary point $x$ of $E$ and does not intersect set $E$ in some neighborhood $U$ of $x$, i.e. $\gamma \cap E \cap U=\varnothing$. Notice that $\gamma$ cuts the plane into two halfplanes. Let $P$ be the half-plane such that $P \cap U \cap E=\varnothing$. We say that a ray $\eta_{x}$ starting at $x$ lies above the straight line $\gamma$ if $\eta_{x} \subset P$.

Lemma 2.16. Suppose an open bounded set $E \subset \mathbb{R}^{2}$ has $Z$-property and consists of three components. If $\partial E$ is smooth, then $E$ has three distinct inner supporting rays starting at a point of 1-nonsemiconvexity of $E$ and 
each of them is inner supporting for $E$ with respect to a unique component of the set.

Proof. Let $E_{j}, j=1,2,3$, be components of $E$ and let $x \in \mathbb{R}^{2} \backslash \bar{E}$ be a point of 1-nonsemiconvexity of the set. By Lemma 2.8 each $E_{j}, j=1,2,3$, has exactly two supporting rays which we denote by $\xi_{x}^{j, 1}, \xi_{x}^{j, 2}$, respectively. Notice that in general some of $\xi_{x}^{j, k}, j=1,2,3, k=1,2$, can coincide, see Figure 2.7b).

By Lemma 2.15, none of the components of $E$ is projected on the union of the others from $x$. Then for $i=1,2,3$ there exists a ray $\tau_{x}^{i}$ starting at $x$ and intersecting a unique component $E_{i}$. In other words,

$$
\tau_{x}^{1} \cap E \equiv \tau_{x}^{1} \cap E_{1}, \quad \tau_{x}^{2} \cap E \equiv \tau_{x}^{2} \cap E_{2}, \quad \tau_{x}^{3} \cap E \equiv \tau_{x}^{3} \cap E_{3} .
$$

Then the rays $\tau_{x}^{1}, \tau_{x}^{2}$ and $\tau_{x}^{3}$ cut the plane into three open components $G_{1}$, $G_{2}, G_{3}$ such that

$$
\partial G_{1}=\tau_{x}^{2} \cup \tau_{x}^{3}, \quad \partial G_{2}=\tau_{x}^{3} \cup \tau_{x}^{1}, \quad \partial G_{3}=\tau_{x}^{1} \cup \tau_{x}^{2} .
$$

Consider the closure of the domain $G_{3}$ between the rays $\tau_{x}^{1}, \tau_{x}^{2}$. We claim that $\bar{G}_{3}$ does not contain points of $E_{3}$. Indeed, $\partial G_{3} \cap E_{3}=\varnothing$ and $\partial G_{3}$ cuts $\mathbb{R}^{2} \backslash\{x\}$ into two components: $G_{3}$ and $G_{2} \cup G_{1} \cup \tau_{x}^{3}$. Since $E_{3}$ is connected, it must be completely contained in one of those components. Hence $E_{3} \cap \tau_{x}^{3} \neq \varnothing$ implies that $E_{3} \subset G_{2} \cup G_{1} \cup \tau_{x}^{3}$.

Thus, the union of rays starting at $x$ and intersecting both $E_{1}, E_{2}$, is open and connected in $\bar{G}_{3}$ and its boundary consists of one ray supporting for $E_{1}$ and one ray supporting for $E_{2}$. Without loss of generality, suppose $\xi_{x}^{1,1}, \xi_{x}^{2,2} \subset \bar{G}_{3}$. Analogically, $\xi_{x}^{2,1}, \xi_{x}^{3,2} \subset \bar{G}_{1}, \xi_{x}^{3,1}, \xi_{x}^{1,2} \subset \bar{G}_{2}$.

Now we prove that for a fixed $k=1,2,3$ one and only one of rays $\xi_{x}^{i, 1}, \xi_{x}^{j, 2} \subset \bar{G}_{k},(i, j, k)=(1,2,3),(2,3,1),(3,1,2)$, is inner supporting for $E$. It is sufficient to prove this for $k=3$. Moreover, due to Lemma 2.11, it suffices to show that one and only one of rays $\xi_{x}^{1,1}, \xi_{x}^{2,2}$ is inner supporting for the set $E_{1} \cup E_{2}$.

By Lemma 2.5, $\xi_{x}^{j, j} \cap \partial E_{j} \neq \varnothing, \xi_{x}^{j, j} \cap E_{j}=\varnothing, j=1,2$. Consider the points $y_{j} \in \xi_{x}^{j, j} \cap \partial E_{j}, j=1,2$. By smoothness of $\partial E$ and since $\xi_{x}^{j, j} \cap E_{j}=\varnothing, j=1,2$, the rays $\xi_{x}^{1,1}, \xi_{x}^{2,2}$ are tangent to $\partial E_{1}, \partial E_{2}$ at the points $y_{1}, y_{2}$, respectively. Without loss of generality, suppose that there are points $b_{1}, b_{2} \in \xi_{x}^{1} \cap E_{2}$ such that $\left|b_{1}-x\right|<\left|y_{1}-x\right|<\left|b_{2}-x\right|$, see Figure 2.6a). Since ray $\xi_{x}^{1,1}$ is tangent to $\partial E_{1}$ at $y_{1}$, all rays starting at $y_{1}$ and not intersecting $E$ should lie above the straight containing $\xi_{x}^{1,1}$. On the other hand, all these rays intersect any curve in $E_{2}$ connecting points $b_{1}, b_{2}$, as $E_{2}$ is connected, which gives that these rays intersect $E_{2}$ and 
therefore $y_{1}$ is a point of 1 -nonsemiconvexity of $E$. This contradicts weakly 1-semiconvexity of the set.

Thus, $|b-x|<\left|y_{1}-x\right|$ or $|b-x|>\left|y_{1}-x\right|$ for any point $b \in \xi_{x}^{1,1} \cap E_{2}$. These statements are also true for the ray $\xi_{x}^{2,2}$. Furthermore, $\left|b_{1}-x\right|>\left|y_{2}-x\right|$ for any point $b_{1} \in \xi_{x}^{2} \cap E_{1}$ if and only if $\left|b_{2}-x\right|<\left|y_{1}-x\right|$ for any point $b_{2} \in \xi_{x}^{1,1} \cap E_{2}$. Swapping indices we will also get that $\left|b_{2}-x\right|>\left|y_{1}-x\right|$ for any point $b_{2} \in \xi_{x}^{1,1} \cap E_{2}$ if and only if $\left|b_{1}-x\right|<\left|y_{2}-x\right|$ for any point $b_{1} \in \xi_{x}^{2,2} \cap E_{1}$, see Figure 2.6b). Otherwise, $E_{1}, E_{2}$ would be overlapping.

Hence, $\xi_{x}^{j, j}$ is supporting for $E_{j}$ and $\xi_{x}^{j, j} \cap\left(\left(E_{1} \cup E_{2}\right) \backslash E_{j}\right) \neq \varnothing, j=1,2$, but $|b-x|>\left|y_{j}-x\right|$ for each $y_{j} \in \partial E_{j}$ and each $b \in \xi_{x}^{j, j} \cap\left(\left(E_{1} \cup E_{2}\right) \backslash E_{j}\right)$, if $j=1$ or $j=2$, which was necessary to prove.

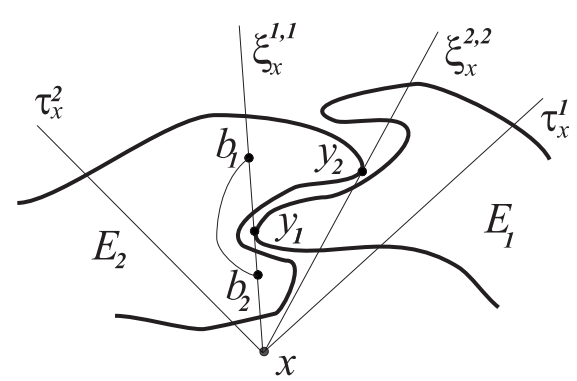

a)

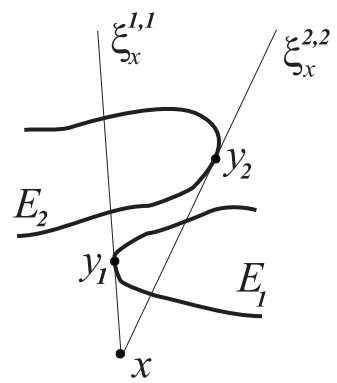

b)

FiguRE 2.6.

Thus, $E$ has no more than three inner supporting rays which we denote by $\xi_{x}^{k}, i=1,2,3$, such that $\xi_{x}^{k} \subset \bar{G}_{k}$. Herewith, if $\xi_{x}^{k} \subset G_{k}, k=1,2,3$, then the ray $\xi_{x}^{k}$ is supporting for a unique of components $E_{j}, j=1,2,3$, $j \neq k$, and therefore, it is inner supporting for $E$ with respect to a unique component of $E$.

Since each $\bar{G}_{k}$ contains only points of $E_{i}, E_{j}$ for

$$
(i, j, k)=(1,2,3),(2,3,1),(3,1,2),
$$

neither of the rays $\xi_{x}^{k} \subset \bar{G}_{k}, k=1,2,3$, can be inner supporting with respect to three components $E_{j}, j=1,2,3$, at the same time.

Since $\bar{G}_{i} \cap \bar{G}_{j}=\tau_{x}^{k},(i, j, k)=(1,2,3),(2,3,1),(3,1,2)$, a ray $\xi_{x}^{k} \subset \bar{G}_{k}$, $k=1,2,3$, can be inner supporting with respect to two components if and only if it coincides with a neighboring inner supporting ray on the common boundary of the respective sets $G_{j}, j=1,2,3$. Let us consider this case and prove that it is not possible under the lemma conditions. By this we will show that $E$ has not less than three inner supporting rays starting at 
$x$ and $\xi_{x}^{k}$ is inner supporting for $E$ with respect to a single component of the set in $\partial G_{k}, k=1,2,3$, as well.

Without loss of generality, assume that two inner supporting rays $\xi_{x}^{1}, \xi_{x}^{2}$ coincide with the boundary ray $\tau_{x}^{3}$, see Figure 2.7a). By the constructions, $\xi_{x}^{1}$ can be supporting for $E_{2}$ or $E_{3}$. But since $\tau_{x}^{3}$ intersects component $E_{3}$ and $\xi_{x}^{1}$ coincides with $\tau_{x}^{3}$, the ray $\xi_{x}^{1}$ is supporting for $E_{2}$. Similarly, the ray $\xi_{x}^{2}$ is supporting for $E_{1}$.

Let $y_{j}$ be the nearest to $x$ point of $\tau_{x}^{3} \cap \partial E_{j}, j=1,2$. Since $\tau_{x}^{3} \cap E_{j}=\varnothing$, $j=1,2$, and by smoothness of $\partial E$, the ray $\tau_{x}^{3}$ is tangent to $\partial E_{j}$ at the points $y_{j}, j=1,2$. The points $y_{1}, y_{2}$ do not coincide, since otherwise one can not draw a ray starting at the point $y_{1}=y_{2}$ and does not intersect $E$, which contradicts to weak 1-semiconvexity of $E$. Assume for definiteness
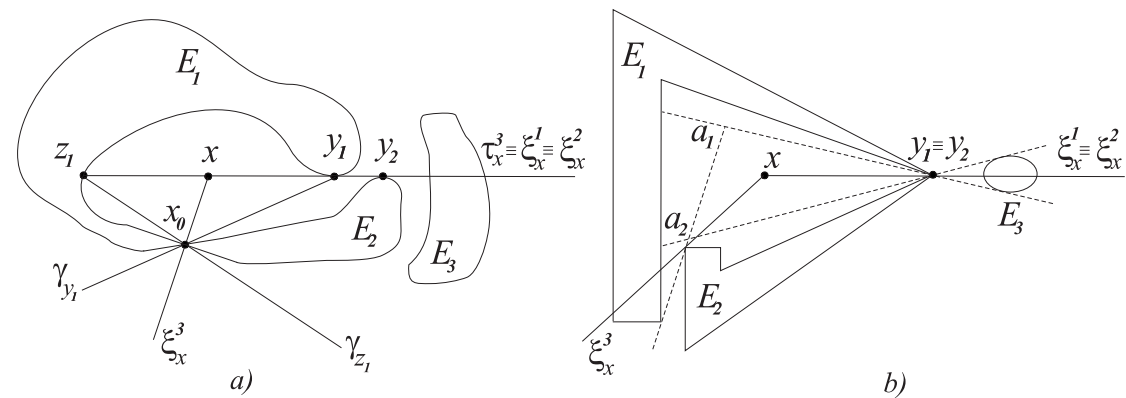

Figure 2.7.

that $x$ is closer to $y_{1}$ than to $y_{2}$, i.e. $\left|x-y_{1}\right|<\left|x-y_{2}\right|$. Then, let us draw any ray $\gamma_{y_{1}}$ starting at $y_{1}$ and not intersecting $E$. It lies above the straight line containing the ray $\tau_{x}^{3}$, since $\partial E$ is smooth. The ray, complementary to $\tau_{x}^{3}$, intersects $\partial E$ at the point $z_{1}$ which is nearest to $x$ along this ray.

Let us draw a ray $\gamma_{z_{1}}$ starting at $z_{1}$ and also not intersecting $E$. By Lemma 2.12, among all rays starting at point $x$ and crossing rays $\gamma_{y_{1}}$ and $\gamma_{z_{1}}$ there exist two inner supporting rays different from $\tau_{x}^{3}$. If they are distinct, this contradicts to the fact that $E$ has only two inner supporting rays by our assumption. Such an inner supporting ray is unique only if rays $\gamma_{y_{1}}$ and $\gamma_{z_{1}}$ are intersecting and the inner supporting ray $\xi_{x}^{3}$ passes through the point $x_{0} \in \gamma_{y_{1}} \cap \gamma_{z_{1}}$.

Since the polygonal chain $\gamma_{y_{1}} \cup\left\{y_{1}\right\} \cup y_{1} z_{1} \cup\left\{z_{1}\right\} \cup \gamma_{z_{1}}$ is self-intersecting, it cuts the plane into three components (parts). The first part contained inside the $\triangle y_{1} z_{1} x_{0}$ does not have points of $E$, by condition 3) of Lemma 2.12. Since the ray $\tau_{y_{1}}^{3} \subset \tau_{x}^{3}$ is such that $y_{1} \in \tau_{y_{1}}^{3} \cap \partial E_{1}, y_{2} \in \tau_{y_{1}}^{3} \cap \partial E_{2}$, and $\tau_{y_{1}}^{3} \cap E_{3} \neq \varnothing$, the second part of the plane that holds $\tau_{y_{1}}^{3}$ contains all three components of $E$. Thus, the third part also does not have points 
of $E$, which allows the ray $\xi_{x}^{3}$ to not intersect $E$. This contradicts to non 1-semiconvexity of $E$. Lemma is proved.

Remark 2.17. Lemma 2.16 fails for sets with non-smooth boundary. Figure 2.7b) contains an example of open bounded set having $Z$-property and consisting of three components which has only two inner supporting rays starting at a point of 1-nonsemiconvexity $x \in \operatorname{Int}\left(\triangle y_{1} a_{1} a_{2}\right)$.

In the following lemmas we will assume that inner supporting rays of the set start at some point of its 1-nonsemiconvexity.

Lemma 2.18. There exists no open bounded subset $E \subset \mathbb{R}$ with smooth boundary having $Z$-property, consisting of three components $E_{1}, E_{2}, E_{3}$, and such that two of inner supporting rays of $E$ are inner supporting with respect to the same component.

Proof. Let us prove the lemma by the contradiction. Let $x \in \mathbb{R}^{2} \backslash \bar{E}$ be a point of 1-nonsemiconvexity of $E$. By Lemma 2.16, $E$ has three inner supporting rays $\xi_{x}^{i}, i=1,2,3$, and each of them is inner supporting with respect to a unique component of $E$.

Without loss of generality, suppose that the rays $\xi_{x}^{1}$ and $\xi_{x}^{2}$ are inner supporting for $E$ with respect to the component $E_{1}$ and $\xi_{x}^{3}$ is inner supporting with respect to $E_{3}$.

Consider the intersections $\xi_{x}^{1} \cap \partial E_{1}, \xi_{x}^{2} \cap \partial E_{1}, \xi_{x}^{3} \cap \partial E_{3}$. By Lemma 2.5, these intersections are not empty. Let $y_{1} \in \xi_{x}^{1} \cap \partial E_{1}, y_{2} \in \xi_{x}^{2} \cap \partial E_{1}, y_{3} \in$ $\xi_{x}^{3} \cap \partial E_{3}$ be the nearest points to $x$. Since $\xi_{x}^{i}, i=1,2,3$, does not intersect the component for which it is supporting and by smoothness of $\partial E$, it follows that $\xi_{x}^{i}$ is tangent to the boundary of the correspondent component at the point $y_{i}, i=1,2,3$. This implies that all rays starting at $y_{i}$ and not intersecting $E$ lie above the straight line containing the supporting ray $\xi_{x}^{i}$.

Let $S$ be the sector between rays $\xi_{x}^{1}$ and $\xi_{x}^{2}$ containing the component $E_{1}$. We claim that then the ray $\xi_{x}^{3}$ is not contained in $S$. Indeed, otherwise $E_{3}$ would be contained in the open component of $\mathbb{R}^{2} \backslash E$ bounded by the intervals $x y_{1}, x y_{2}$ and the part of $\partial E_{1}$ between points $y_{1}, y_{2}$. Then $E_{3}$ would be projected on $E_{1}$, which contradicts to Lemma 2.15. Therefore $\xi_{x}^{3}$ is contained in the open sector that is complementary to $S$.

Let $\alpha$ be the angle of sector $S$. Consider two cases: a) $\alpha \geqslant \pi$ and b) $\alpha<\pi$.

a) Let $z_{3} \in \partial E$ be the point contained in the ray complementary to $\xi_{x}^{3}$ and closest to $x$. Then $z_{3} \in \partial E_{1}$, see Figure 2.8. Let us draw a ray $\gamma_{z_{3}}$ that does not intersect $E$. It passes through the interval $x y_{1}$ or $x y_{2}$. It is sufficient to consider the case with interval $x y_{1}$. For the interval $x y_{2}$, the arguments will be the same. 


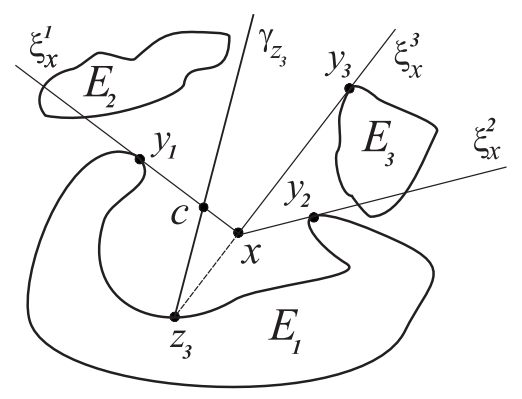

FiguRE 2.8.

By Lemma 2.12, one of the rays $\xi_{x}^{i}, i=1,2,3$, satisfies conditions 1 )-3) of that lemma for the ray $\gamma_{z_{3}}$. Denote that ray by $\xi_{x}$.

Since $\xi_{x}^{2}$ lies in the half-plane not containing $\gamma_{z_{3}}$, the ray $\xi_{x}^{2}$ does not intersect $\gamma_{z_{3}}$ and therefore $\xi_{x} \neq \xi_{x}^{2}$. As $\gamma_{z_{3}} \nsubseteq \xi_{x}^{3}$, the ray $\xi_{x}^{3}$ does not intersect ray $\gamma_{z_{3}}$ as well and therefore $\xi_{x} \neq \xi_{x}^{3}$.

Let $c=\xi_{x}^{1} \cap \gamma_{z_{3}}$. Since $\partial E$ is smooth and $z_{3}$ belongs to the open part of $\partial E_{1}$ between points $y_{1}$ and $y_{2}$, it follows that the point $y_{1} \in \partial E_{1}$ satisfies the inequality $\left|y_{1}-x\right|>|c-x|$ and therefore condition 2) of Lemma 2.12 is not fulfilled for the ray $\xi_{x}^{1}$. Thus, $\xi_{x} \neq \xi_{x}^{1}$ as well.

We get a contradiction, whence our assumption is incorrect for $\alpha \geqslant \pi$.

b) Suppose $\alpha<\pi$. If the inner supporting ray $\xi_{x}^{3}$ is contained in the open sector $S^{\prime}$ between the rays complementary to $\xi_{x}^{1}$ and $\xi_{x}^{2}$, then $z_{3} \in \partial E_{1}$ and the arguments should be as in the case a). Thus, we again get a contradiction.

Let $\alpha_{1}\left(\operatorname{resp} \alpha_{2}\right)$ be the angle between nearest-neighbor rays $\xi_{x}^{3}$ and $\xi_{x}^{1}$ (resp. $\xi_{x}^{3}$ and $\xi_{x}^{2}$ ), see Figure 2.9. Suppose $\xi_{x}^{3}$ is not contained in the sector $S^{\prime}$. Then $\alpha_{1} \neq \alpha_{2}$.

For definiteness assume that $\alpha_{1}>\alpha_{2}$ and $\alpha_{1} \geqslant \pi$. By smoothness of $\partial E$, the ray $\gamma_{y_{1}}$ that does not intersect $E$ should lie above the straight that contains $\xi_{x}^{1}$. But all such rays intersect $E$. Indeed, if one assumes that there exists a ray $\gamma_{y_{1}}$ not intersecting $E$, then by Lemma 2.12, one of the inner supporting rays $\xi_{x}^{j}, j=1,2,3$, intersects $\gamma_{y_{1}}$. However the ray $\xi_{x}^{1}$ can intersect $\gamma_{y_{1}}$ only if $\gamma_{y_{1}} \subset \xi_{x}^{1}$, which is not possible, since $\xi_{x}^{1}$ intersects $E$ and $\gamma_{y_{1}}$ does not. By the constructions, the rays $\xi_{x}^{3}$ and $\xi_{x}^{2}$ lie in the half-plane different from the one that contains $\gamma_{y_{1}}$. Then the rays $\xi_{x}^{3}, \xi_{x}^{2}$ also do not intersect $\gamma_{y_{1}}$.

Thus, $y_{1}$ is a point of 1 -nonsemiconvexity of $E$, which contradicts to its weak 1-semiconvexity. We have now reached a contradiction and our assumption is wrong for $\alpha<\pi$ as well. Lemma is proved. 


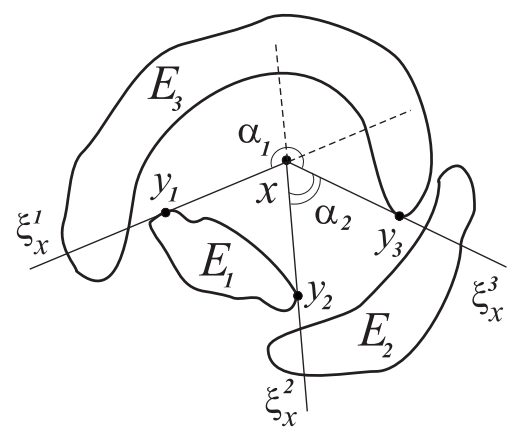

FiguRE 2.9 .

Lemma 2.18 fails for sets with non-smooth boundary as $\alpha<\pi$, see Figure 2.1b).

Lemma 2.19. There exists no open bounded set with smooth boundary in the plane having $Z$-property, consisting of three components and such that there is a bijection between the inner supporting rays of the set and the components with respect to which they are inner supporting.

Proof. Let us prove the lemma by the contradiction. Suppose set $E \subset \mathbb{R}^{2}$ is open, bounded, having $Z$-property, and consisting of three components $E_{j}, j=1,2,3$. Let $x \in \mathbb{R}^{2} \backslash \bar{E}$ be a point of 1-nonsemiconvexity of $E$. By Lemma 2.16, $E$ has three inner supporting rays $\xi_{x}^{i}, i=1,2,3$. Thus, without loss of generality, suppose $\xi_{x}^{i}$ is inner supporting for $E$ with respect to $E_{i}, i=1,2,3$.

Let $y_{i} \in \xi_{x}^{i} \cap \partial E_{i}$ be the nearest point of $\xi_{x}^{i} \cap \partial E_{i} \neq \varnothing$ to $x$, Figure 2.10a). Since neither of the rays $\xi_{x}^{i}, i=1,2,3$, intersect the respective component $E_{i}$ for which it is supporting, it follows from smoothness of $\partial E$ that each ray $\xi_{x}^{i}$ is tangent to $\partial E_{i}$ at the point $y_{i}$.

By Lemma 2.8, each component $E_{i}, i=1,2,3$, is contained in the respective sector between two supporting rays of $\angle \alpha_{i}>0, i=1,2,3$. Without loss of generality, let us consider the polar coordinate system $(\varphi, \rho)$, where point $x$ is the pole, inner supporting ray $\xi_{x}^{1}$ is the polar axis, and $\eta_{x}(\varphi)$ is a ray starting at $x$ and generating an angle $\varphi$ with ray $\xi_{x}^{1}$, see Figure 2.10. Since $E$ is weakly 1 -semiconvex, there exists a ray $\gamma_{y_{1}}$ not intersecting $E$. As $\partial E_{1}$ is smooth, the ray $\gamma_{y_{1}}$ should lie above the straight that contains ray $\xi_{x}^{1}$. Let us chose a positive angular coordinate determined by a ray starting at $x$ and intersecting ray $\gamma_{y_{1}}$. In this coordinate system, let angle $\phi$ be such that $\eta_{x}(\phi) \| \gamma_{y_{1}}, 0<\phi<\pi$. 
By Lemma 2.12 , for the ray $\gamma_{y_{1}}$ there exists an inner supporting ray $\xi_{x}$ satisfying conditions 1)-3) of that lemma. Since $E$ has only three inner supporting rays, one of rays $\xi_{x}^{i}, i=1,2,3$, coincides with $\xi_{x}$.

Since $\partial E$ is smooth, the triangle $x y_{1} c$, where $c=\xi_{x} \cap \gamma_{y_{1}}$, does not degenerate into the interval $x y_{1}$. Then $\xi_{x} \neq \xi_{x}^{1}$, which gives $\xi_{x}=\xi_{x}^{2}$ or $\xi_{x}=\xi_{x}^{3}$.

Without loss of generality suppose that $\xi_{x}=\xi_{x}^{2}$. Then by Lemma 2.12, $\xi_{x}^{2}=\eta_{x}\left(\varphi_{2}\right), 0<\varphi_{2}<\phi<\pi$, and

1) $\xi_{x}^{2} \cap \gamma_{y_{1}}=c\left(\varphi_{2}\right)$;

2) $y_{2} \in x c\left(\varphi_{2}\right)$;

3) $E \cap \operatorname{Int}\left(\triangle x y_{1} c\left(\varphi_{2}\right)\right)=\varnothing$.

This implies that, in a certain coordinate system, the component $E_{2}$ is contained in the angle that corresponds to the interval $\left(\varphi_{2}, \varphi_{2}+\alpha_{2}\right)$ between its two supporting rays. Then $E_{1}$ is contained in the angle $\left(2 \pi-\alpha_{1}, 2 \pi\right)$.

If $\varphi_{2}+\alpha_{2}>2 \pi-\alpha_{1}$, then $\left(\varphi_{2}, \varphi_{2}+\alpha_{2}\right) \cap\left(2 \pi-\alpha_{1}, 2 \pi\right) \neq \varnothing$, see Figure 2.10. Hence neither of these intervals is completely contained in the other one, otherwise one of the components $E_{1}, E_{2}$ would be projected on the other. Consider the rays $\tau_{x}^{1}, \tau_{x}^{2}$ from the proof of Lemma 2.16. They are contained in the angle $\left(\varphi_{2}, \varphi_{2}+\alpha_{2}\right) \cup\left(2 \pi-\alpha_{1}, 2 \pi\right)=\left(\varphi_{2}, 2 \pi\right)$.

Let $\left[\varphi_{3}, \varphi_{4}\right] \subset\left(\varphi_{2}, 2 \pi\right)$ be the angle between rays $\tau_{x}^{1}, \tau_{x}^{2}$. The component $E_{3}$ is not contained in $\left[\varphi_{3}, \varphi_{4}\right]$, otherwise $E_{3}$ would be projected on $E_{1} \cup E_{2}$. Thus, by the proof of Lemma 2.16, the third inner supporting ray $\xi_{x}^{3}$ is contained in $\left[\varphi_{3}, \varphi_{4}\right]$ and is inner supporting for $E$ with respect to one of the components $E_{1}, E_{2}$. This contradicts to our assumption that $\xi_{x}^{3}$ is inner supporting with respect to the component $E_{3}$.

If $\varphi_{2}+\alpha_{2} \leqslant 2 \pi-\alpha_{1}$, then either angle $\left[\varphi_{2}+\alpha_{2}, 2 \pi-\alpha_{1}\right]$ or $\left[0, \varphi_{2}\right]$ does not contain points of $E_{3}$ and therefore points of $E$, otherwise, component $E_{1}$ would be projected on $E_{3}$. But this gives that we can draw a ray starting at $x$ and not intersecting set $E$, which contradicts to non 1 -semiconvexity of $E$. Thus the assumption is wrong. Lemma is proved.

Lemma 2.19 also fails for sets with non-smooth boundary, see Figure 2.3b).

\section{Proof of Theorem 1.8}

By Theorem 1.6, the set $E$ does not consist of one or two components. Due to Example 2.4, it suffices to prove that the set does not consist of three components as well. Let us prove this by contradiction. So, suppose $E$ consists of three connected components $E_{i}, i=1,2,3$.

Since $E$ is not 1-semiconvex, it follows that there exists a point of 1nonsemiconvexity $x \in \mathbb{R}^{2} \backslash \bar{E}$ of $E$ (since $E$ is a weakly 1-semiconvex set, we do not consider points of $\partial E$ as points of 1-nonsemiconvexity of $E$ ). 


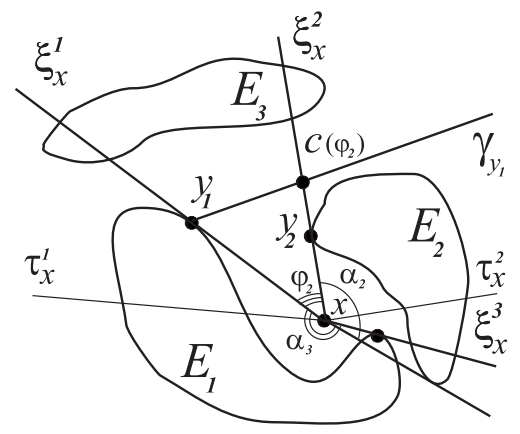

Figure 2.10.

By Lemma 2.16, $E$ has three inner supporting rays $\xi_{x}^{i}, i=1,2,3$, starting at $x$, and each of them is inner supporting for $E$ with respect to a single component of the set. In other words, there is a function

$$
f:\left\{\xi_{x}^{1}, \xi_{x}^{2}, \xi_{x}^{3}\right\} \longrightarrow\left\{E_{1}, E_{2}, E_{3}\right\} .
$$

Since by Lemma 2.8 each component has at most two supporting rays, there possible exactly two cases:

I) $f$ is a bijection, i.e., each ray $\xi_{x}^{k}, i=1,2,3$, is inner supporting for $E$ with respect to one and only one component of $E$, Figure $3.1 \mathrm{a}$;

II) there exist $\xi_{x}^{k}, \xi_{x}^{l}, k, l=1,2,3, k \neq l$, such that $f\left(\xi_{x}^{k}\right)=f\left(\xi_{x}^{l}\right)$, i.e., two rays of $\xi_{x}^{k}, i=1,2,3$, are inner supporting for $E$ with respect to the same its component and the third is inner supporting for $E$ with respect to one of the other two components, Figure 3.1b).

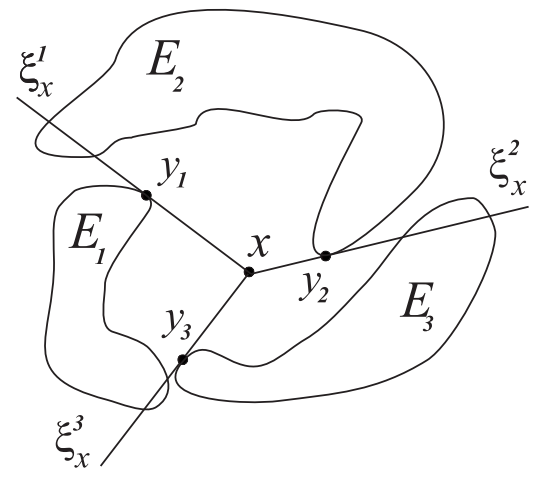

a)

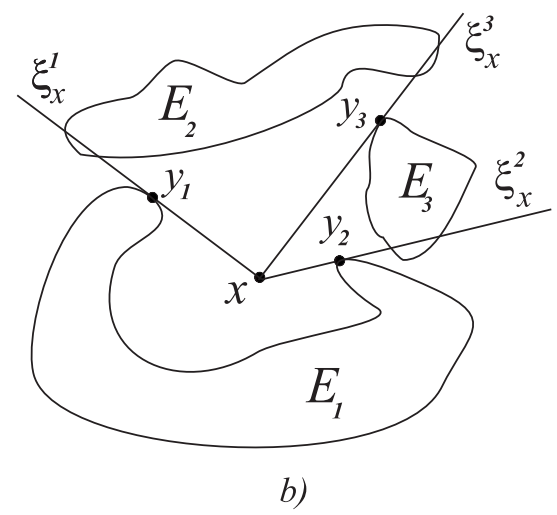

FiguRE 3.1.

Then the case II) is not possible due to Lemma 2.18 while the case I) is not possible by Lemma 2.19. Therefore the assumption that the set 
consists of three components is incorrect. Example 2.4 completes the proof of Theorem 1.8.

Corollary 3.1. Suppose an open bounded subset $E \subset \mathbb{R}^{2}$ has $Z$-property and consists of four components with smooth boundary. Then none of its components is projected on the union of the others from a point of 1-nonsemiconvexity of $E$.

Proof. The proof is similar to the proof of Lemma 2.15. Let $E_{i}, i=\overline{1,4}$, be components of $E$, and $x \in \mathbb{R}^{2} \backslash \bar{E}$ be a point of 1-nonsemiconvexity of $E$. Without loss of generality, suppose that $E_{1}$ is projected from $x$ on $\underset{i=2}{4} E_{i}$. Then the set, consisting only of components $E_{i}, i=2,3,4$, has $Z$-property, which contradicts to Theorem 1.8.

Acknowledgements. The author was supported by the grant of the President of Ukraine for competitive projects F75/30308 of the State Fund for Fundamental Research.

\section{REFERENCES}

[1] L. A. Aizenberg. Linear convexity in $\mathbb{C}^{n}$ and the separation of singularities of holomorphic functions. Bull. Acad. Pol. Sci., 15(7):487-495, 1967.

[2] L. A. Aizenberg. The expansion of holomorphic functions of several complex variables in partial fractions. Sibirsk. Mat. Ž., 8:1124-1142, 1967.

[3] H. K. Dakhil. The shadows problems and mappings of fixed multiplicity. PhD, Institute of Mathematics of NAS of Ukraine, Kyiv, 2017 (in Ukrainian).

[4] G. Khudaiberganov. On the homogeneous polynomially convex hull of a union of balls, volume Manuscr.dep. 21.02.1982 of 85 Dep. M.: VINITI, 1982 (in Russian).

[5] A. Martineau. Sur la topologie des espaces de fonctions holomorphes. Math. Ann., 163(1):62-88, 1966 .

[6] B. A. Rozenfeld. Multi-dimensional spaces. Moskow, 1966 (in Russian).

[7] Yu. B. Zelinskii. Generalized convex envelopes of sets and the problem of shadow. $J$. Math. Sci. (N.Y.), 211(5):710-717, 2015, doi: 10.1007/s10958-015-2626-8. Translation of Ukr. Mat. Visn. 12 (2015), no. 2, 278-289.

[8] Yu. B. Zelinskii. Variations to the problem of "shadow". Zbirn. Prats Inst. Math. NANU, 14(1):163-170, 2017 (in Ukrainian).

[9] Yu. B. Zelinskii, I. Yu. Vygovskaya, M. V. Stefanchuk. Generalized convex sets and a shadow problem. Ukraïn. Mat. Zh., 67(12):1658-1666, 2015, doi: $10.1007 / \mathrm{s} 11253-016-1196-3$.

Received: November 17, 2017, accepted: November 2, 2019.

Tetiana Osipchuk

Institute of Mathematics of NAS of Ukraine, Tereschenkivska Str., 3, Kyiv, 01024, UKRAINE

Email: osipchuk.tania@gmail.com 